\title{
A Study on the Causes for Failures in Mathematics by Engineering Students using Combined Fuzzy Clustering Model (CFCLM)
}

\author{
A. Praveen Prakash \\ Department of Mathematics \\ Hindustan University \\ Padur, Chennai - 603103
}

\author{
J. Esther Jerlin \\ Department of Mathematics \\ Hindustan University \\ Padur, Chennai - 603103
}

\author{
J. Bennilo Fernandes \\ M.Tech, Embedded Systems \\ Hindustan University \\ Padur, Chennai - 603103
}

\begin{abstract}
The main objective of this paper is to introduce a new fuzzy model called Combined Fuzzy Clustering Model(CFCLM). First time the working rule for this model is derived by creating an algorithm. The study of failure in Mathematics among engineering students is analyzed using Combined Fuzzy Clustering (CFCLM) model. It is important to note that the performance in mathematics in all the engineering colleges, including IITs by students undergoing B.E and B.Tech courses are considerably poor. This paper analyzes the problem by taking a pilot survey from 100 Engineering students, Engineering faculties and parents of engineering students. This paper consists of eight sections. Section one is introductory in nature that gives the background of the problem under study and gives the justification for having chosen to use the "Combined Fuzzy Clustering Model" approach to seek the dominant cause for the failure. Section two discuss about the causes for failure in mathematics by engineering students.Section three gives the preliminaries and the basics of Combined Fuzzy Clustering Model approach. Section fourdeals with the application of the said approach in determining the cluster of problems, that fall under the three categories viz, 'low', 'moderate' and 'high'. Section five consists of the algorithm used for the study of the problem. Sixth and Seventh section give the main findings as conclusion and suggestions.And, the final eighth section gives the references used for the study.
\end{abstract}

\section{Keywords}

Clustering, Fuzzy Clustering, Combined Fuzzy ClusteringModel (CFCLM),Degrees of Membership, Failure in Maths.

\section{INTRODUCTION}

Mathematics offers effective tools to solve problems posed in the field of Engineering. "The past decade has seen a serious decline in students' basic mathematical skills and level of preparation on entry into higher education... many students..embark on engineering degree programmes without the necessary maths skills required for the course.." The quote is from the website of Engineering Subject Centre, a national center delivering support for learning and teaching in the U.K higher education community. The statement is universally applicable.

In Tamil Nadu, by 1984, there was just six Government Engineering Colleges and a handful of ITIs and polytechnics. From 1985 onwards the Engineering education was a period to private bodies. Ever since, the Engineering education was opened to private parties, Businessmen, politicians started opening Engineering colleges. The number of colleges grew in geometric proportion and as a result Tamil Nadu has over 570 Engineering Institution. As the quantity increased the quality started declining.The problem of most I year B.E/B.Tech students failing in 'Engineering Mathematics' seems to be thegrowing problem at present. The reasons for high failure rate varies from individual to individual. The learners coming to engineering from diverse backgrounds, lack of proper basics in learning the subject by applying or problem-solving methods, shortage of good mathematics faculties in Engineering Colleges and joining the students not out of their interest but out of compulsion from parents are the major causes for such problems.

\section{METHODOLOGY}

In this methodology, the causes for failures in mathematics among students studying Engineering courses in collegesare categorized as twenty five attributes by speaking and collecting the causes from engineering students, engineering faculties and to the parents of engineering students. The following twenty five attributes of causes for the failuresare arrived at by interviewing engineering students of all four years, engineering faculties and parents of engineering students in Chennai.

1. Teachers lack knowledge in teaching methodology

2. Parents join under compulsion in Engineering

3. Students are not regular to classes

4. Higher secondary schools fail to provide clear knowledge in basics of XI and XII std syllabus

5. Harsh approach and giving rude punishment by teachers

6. Students don't have interest and involvement in maths

7. Teachers are not dedicated to teaching profession

8. Institution fails to provide good atmosphere innurturing teacher-student relation

9. Lack of sufficient knowledge in basics by students

10. Lack of sufficient practice by students

11. Just enter the college with the attitude of enjoying college life

12. Problem of language to understand mathematical terminology and the basics

13. Lack of problem solving skill

14. Memory loss for the students 
15. Teachers fail to assess the students continuously by keeping test, assignments etc

16. Lack of Logical reasoning and application skill

17. Lack of concentration by students

18. Providing only notes as study material than teaching

19. Problem of health and nutrition

20. Preconceived notion that mathsis difficult subject and having fear for it

21. Lack of confidence especially for this subject Maths

22. Peer-group pressure

23. Considering arrear as fashion

24. Lack of interest in planning the time

25. Childhood aversion to maths due to varied environmental conditions

These attributes that define the major causes for student's failure in Mathematics are summarized based on the linguistic questionnaire administered to students, teachers, parents and members of management. Fuzzy c- means clustering is done using (5.1), to classify the causes under three categories viz, low, medium and high.

\section{PRELIMINARIES}

\subsection{Hard Clustering}

Hard Clustering consists of hard partition of the data set $\mathbf{Z}$. In other words, the data set $\mathrm{Z}$ is divided into $c \geq 2$ cluster which means,

$\bigcup_{i=1}^{c} A_{i}=Z$

and $A_{i} \cap A_{j}=\phi, \forall \mathrm{i} \neq \mathrm{j}$

Also, none of the sets, $A_{i}$ may be empty. To indicate a partioning, the membership functions $\mu_{\mathrm{k}}(x)$ is applied. If $\mu_{\mathrm{k}}(x)=1$, then object $x$ isincluster $k$.Based onthemembership functions, the PartitionMatrix Uis assembled,ofwhich $\mu_{\mathrm{k}}(x)$ aretheelements. Finallythereisa rulethat $\forall \mathrm{x}$,

$\sum_{i=1}^{c} ?_{k}(x)=1 \quad \forall x$

In otherwords, every object is only part of one cluster.

\subsection{Fuzzy Clustering}

Hard clustering has a downside. When an object roughly falls between two clusters $A_{i}$ and $A_{j}$, it has to be put into one of these clusters. Also, outliers have to be put in some cluster. This is undesirable. Butitcanbefixedbyfuzzyclustering.[2],[3].

In Fuzzy clustering, Fuzzy partition of the data is made. Now, the membership function $\mu_{\mathrm{k}}(\mathrm{x})$ can be any value between 0 and 1. This means that an object $\mathrm{Z}_{\mathrm{k}}$ can be for 0.2 parts in $\mathrm{Ai}$ and for 0.8 parts in Aj. However, requirement(2) still applies. So, the sum of the membership functions still has to be 1 . The set of all fuzzy partitions that can be formed in this way is denoted by $\mathrm{M}_{\mathrm{fc}}$. Fuzzy partitioning again has a downside. An outlier in the data also to be (being an object that doesn't really belong to any cluster), assigned it to clusters. That is, the sum of its membership functions still must equal one.

\subsection{Fuzzy C-MeansClustering}

In fuzzy clustering, each point has a degree of belonging to clusters, as in fuzzy logic, rather than belonging completely to just one cluster. Thus, points on the edge of a cluster, may be in a cluster to a lesser degree than points in the center of cluster for each point $\mathrm{x}$ there is no coefficient giving the degree of belonging in the $\mathrm{k}^{\text {th }}$ cluster $\mu_{\mathrm{k}}(\mathrm{x})=1$. Usually, the sumofthosecoefficientsisdefinedtobe1.[9]

$\sum_{n=1}$ mn cluster ? $_{k}=1 \quad \forall x$

Withfuzzyc-means, thecentroid ofacluster isthe meanofallpoints, weighted by theirdegree of belongingtothecluster

Center $_{k}=\frac{\sum_{x} \mathrm{\Xi}_{k}(x)^{m} x}{\sum_{x} \mathrm{Q}_{k}(x)^{m}}$

Thedegreeofbelongingis relatedtothe inverseofthedistancetothecluster

$?_{k}(x)=\frac{1}{d\left(\text { Center }_{k}, x\right)}$.

Thenthecoefficients arenormalizedandfuzzyfield witha realparameter $m>1$ sothattheirsumis1. So

$?_{k}(x)=\frac{1}{\sum_{j}\left(\frac{\text { Center }_{k}, x}{\text { Center }_{j}, x}\right)^{2 /(m-1)}}$.

For $m$ equal to 2 , this is equivalent to normalizing the coefficient linearly to make their sum 1 . When $\mathrm{m}$ is close to1,then cluster center closes to the point is given much more weight than the others, and the algorithm is similar to $k$-means.

\subsection{CombinedFuzzy Clustering Model}

Finite number of Fuzzy Clustering can be combined to produce joint effect of all the Fuzzy clustering. [8].Let Table1,

Table2,...., Tablen be the $n$ tables of degree of membership of the attributes of $n$ experts, then the combined Fuzzy Clustering is obtained by adding the corresponding membership value of each attribute of the table.

The Combined Fuzzy Clustering Model (CFCLM) is obtainedas

Table CFCLM = Table 1+Table 2+....Table n

\section{RESULTS AND DISCUSSION}

On interviewing 100 engineering students, engineering faculties and parents of engineering students in Chennai to find what are the causes for the engineering student's failure in mathematics,and listed the following twenty five attributes. Three sets of experts viz, students, teachers and parents were invited to express their viewpoints. The following figures and tables show the result of our analysis. The analysis is carried on a 10-point rating scale.

The ratings and the Standard Deviation of the attributes for the causes of failures in mathematics by engineering students have been subjected to fuzzy c-means clustering using algorithm (5.1) and the following results are shown in Table 2, Table 3 , 
and Table 4according to the expert's opinion. The following tables are obtained for a 3-cluster combination.

The first cluster comprises of the attributes with average rating from 2 to 5 with a mid-value3.5. The second cluster range is from 3 to 8 with a mid-value 5.5 and the third cluster has a range of 7- 10 with a mid-value 8.5.

The first, second and third clusters range indicates the causes of failures in mathematics by engineering students show the low, moderate and high level of the dominant cause. There is overlapping ranges as in characteristic of a fuzzy based cluster.

For the same,3-cluster Range of level of Dominant Cause (i.e)

Table 1. 3-cluster Range of level of Dominant Cause

\begin{tabular}{|c|c|c|c|}
\hline & Cluster 1 & Cluster 2 & Cluster 3 \\
\hline Range & $\mathbf{2 . 0 - 5 . 0}$ & $\mathbf{3 . 0 - 8 . 0}$ & $\mathbf{7 . 0 - 1 0}$ \\
\hline Mid Value & $\mathbf{3 . 5}$ & $\mathbf{5 . 5}$ & $\mathbf{8 . 5}$ \\
\hline Classification & LOW & MEDIUM & HIGH \\
\hline
\end{tabular}

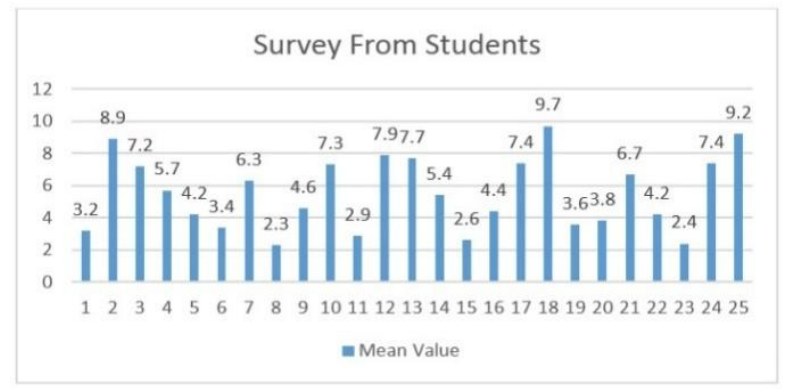

Fig 1: Mean Rating of dominant cause obtained from Engineering students

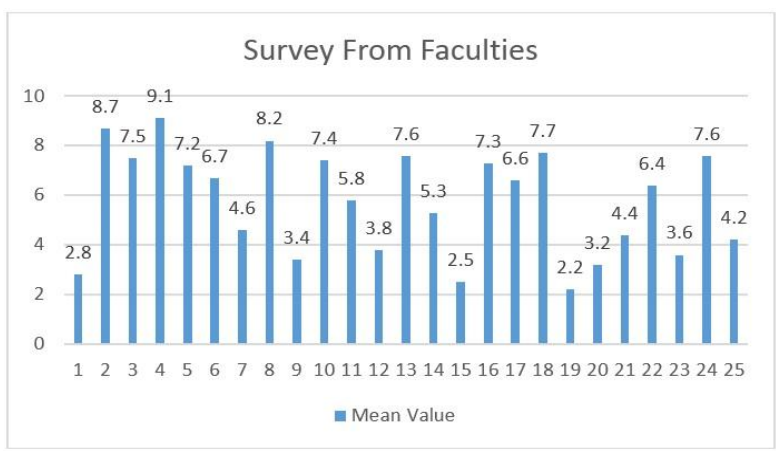

Fig 2: Mean Rating of dominant cause obtained from Engineering Faculties
Survey From Parents

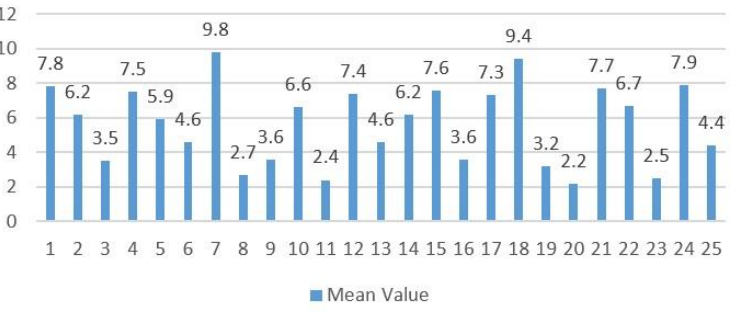

Fig 3: Mean Rating of dominant cause obtained from the Parents of Engineering students

\section{ALGORITHMS}

\subsection{AlgorithmtofindaMembership Values for the Attributes \\ STEP1:Start}

STEP2: Fix, the values of 25 attributes on a 10-pointrating scale in a set D (say)

STEP3: Fix the cluster, which is define das Cluster1=LOW, whose range beginning with 2(bv1) End with5 (ev1). Cluster 2 $=$ MODERATE, whose range beginning with 3(bv2) end with $8(\mathbf{e v 2})$. Cluster3=HIGH, whose range beginning with7(bv3)end with10(ev3).

\section{STEP4:Chooseenelement $x$ inD}

STEP5:If $x<$ ev1, Go toSTEP6, elseGotoSTEP8

STEP 6:If $x>$ bv 2,then $x$ lies in cluster 1and cluster 2 whosemembershipvalueis defined as $\mu_{\mathrm{k}}(\boldsymbol{x})=\operatorname{ev1} \mathbf{x}: \quad x$ bv2,GotoSTEP12, elseGoto STEP7.

STEP7: Then $x$ lies in cluster 1 only, themembershipvalueis $\mu_{\mathrm{k}}(x)=1$ GotoSTEP12

STEP8:If $x<$ ev2GotoSTEP9, elseGotoSTEP11

STEP 9:If $x>b v 3$, then $x$ liesincluster 2and cluster3, whose membershipvalueis definedas $\boldsymbol{\mu}_{\mathrm{k}}(\boldsymbol{x})=\mathbf{e v} 2-\boldsymbol{x}: \boldsymbol{x}$-bv3, GotoSTEP 12, elseGoto STEP10

STEP 10: Then $x$ lies in cluster 2 only, the membership value is $\mu_{\mathrm{k}}(x)=1$ else GotoSTEP11

STEP 11:Then $x$ lies in cluster3only, th e membershipvalueis $\mu_{\mathbf{k}}(x)=1$

STEP12: GotoSTEP 4, untilall thevaluesinDhavebeenchecked STEP13:Stop

Here 'bv' denotes the beginning value and $\mathbf{e v}$ ' denotes the ending the value.[6],[10].

5.2AlgorithmtofindaMembership Values for the AttributesCombined Fuzzy Clustering Model

STEP1:Start

STEP2: $\mathbf{X}=\left(\mathrm{X}_{1}+\mathrm{X}_{2}+\ldots+\mathrm{X}_{\mathrm{n}}\right) / \mathrm{n}$, from the corresponding rows of Table 2,3 \& 4 . GOTO STEP3.

STEP3:L $=\left(\mathrm{L}_{1}+\mathrm{L}_{2}+\ldots+\mathrm{L}_{\mathrm{n}}\right) / \mathrm{n}$, from the corresponding rows of Table 2,3 \& 4 . GOTO STEP4.

STEP4: $\quad \mathbf{M}=\left(\mathrm{M}_{1}+\mathrm{M}_{2}+\ldots+\mathrm{M}_{\mathrm{n}}\right) / \mathrm{n}$, from the corresponding rows of Table 2, 3 \& 4.GOTO STEP5. 
STEP5: $\mathbf{H}=\left(\mathrm{H}_{1}+\mathrm{H}_{2}+\ldots+\mathrm{H}_{\mathrm{n}}\right) / \mathrm{n}$, from the corresponding rows of Table 2,3 \& 4 .GOTO STEP6.

STEP 6: if $(\mathbf{L}<=\mathbf{M})$ and if $(\mathbf{M}<=\mathbf{H})$, put $\mathrm{H}=1$ else $\mathbf{M}=1$, else GOTO $S T E P 7$.

STEP7: if $(\mathbf{L}<=\mathbf{H})$ put $\mathrm{H}=1$ else $\mathrm{L}=1$. GOTO $\boldsymbol{S T E P} 8$.

STEP8: GOTO $S T E P 2$ until attaining all the membership value for the corresponding rows. GOTO STEP9.

\section{STEP 9:Stop}

Here ' $\mathbf{X}$ ' denotes the Mean Value, ' $\mathbf{L}$ ' denotes the LOW, 'M' denotes the Moderate and ' $\mathbf{H}$ ' denotes the High.

\subsection{Method of Hidden Pattern}

Table CFCLM is obtained by calculating

(i) $\quad \mathrm{X}=\frac{\mathrm{X} 1+\mathrm{X} 2+\mathrm{X} 3+\cdots \ldots+\mathrm{Xn}}{n}$

$\mathrm{L}=\frac{\mathrm{L} 1+\mathrm{L} 2+\mathrm{L} 3+\cdots \ldots+\mathrm{Ln}}{n}$

$\mathrm{M}=\frac{\mathrm{M} 1+\mathrm{M} 2+\mathrm{M} 3+\cdots \ldots+\mathrm{Mn}}{n}$

$\mathrm{H}=\frac{\mathrm{H} 1+\mathrm{H} 2+\mathrm{H} 3+\cdots \ldots+\mathrm{Hn}}{n}$

(ii) Among the three clusters, the highest membership value is thresholded and updated as 1 denoting the on state and rest others take the value 0 denoting as off state.[7], [5].

If two clusters have the same membership value then the highest level of classification among them takes the value 1 to denote on state and rest other as 0 to denote off state.
Table 2: Degree of Membership of the attributes Engineering students

\begin{tabular}{|c|c|c|c|c|}
\hline Attributes & Mean $_{1}$ & Low $_{1}$ & Moderate 1 & High $_{1}$ \\
\hline 1 & 3.2 & 0.9 & 0.1 & 0 \\
\hline 2 & 8.9 & 0 & 0 & 1 \\
\hline 3 & 7.2 & 0 & 0.8 & 0.2 \\
\hline 4 & 5.7 & 0 & 1 & 0 \\
\hline 5 & 4.2 & 0.4 & 0.6 & 0 \\
\hline 6 & 3.4 & 0.8 & 0.2 & 0 \\
\hline 7 & 6.3 & 0 & 1 & 0 \\
\hline 8 & 2.3 & 1 & 0 & 0 \\
\hline 9 & 4.6 & 0.2 & 0.8 & 0 \\
\hline 10 & 7.3 & 0 & 0.7 & 0.3 \\
\hline 11 & 2.9 & 1 & 0 & 0 \\
\hline 12 & 7.9 & 0 & 0.1 & 0.9 \\
\hline 13 & 7.7 & 0 & 0.3 & 0.7 \\
\hline 14 & 5.4 & 0 & 1 & 0 \\
\hline 15 & 2.6 & 1 & 0 & 0 \\
\hline 16 & 4.4 & 0.3 & 0.7 & 0 \\
\hline 17 & 7.4 & 0 & 0.6 & 0.4 \\
\hline 18 & 9.7 & 0 & 0 & 1 \\
\hline 19 & 3.6 & 0.7 & 0.3 & 0 \\
\hline 20 & 3.8 & 0.6 & 0.4 & 0 \\
\hline 21 & 6.7 & 0 & 1 & 0 \\
\hline 22 & 4.2 & 0.4 & 0.6 & 0 \\
\hline 23 & 2.4 & 1 & 0 & 0 \\
\hline 24 & 7.4 & 0 & 0.6 & 0.4 \\
\hline 25 & 9.2 & 0 & 0 & 1 \\
\hline
\end{tabular}

Considering engineering students as the experts, degreeofmembership oftheattributesthat refer to the causes of failure in mathematics by engineering students isfoundusingtheabovealgorithm is showninTable:2. Attributes $8,11,15,23$ witha meanrating 2.3,2.9,2.6,2.4 isentirely (100\%)withamembership valueof 1 incluster 1. (i.e.)LOW.

Attributes4, 7, 14, 21withameanrating5.7, 6.3, 5.4, 6.7 isentirely $(100 \%)$ withamembershipvalue of1incluster2.(i.e.)MODERATE.

Attribute $2,18,25$ withameanrating8.9, 9.7, 9.2isentirely $(100 \%)$ with amembershipvalueof 1incluster 3. (i.e.)HIGH.

Attributes 1,5,6,9,16,19,20,22 withameanratings3.2,4.2,3.4,4.6,4. $4,3.6,3.8,4.2$ belongsto $90 \%$ incluster 1 and $10 \%$ incluster $2,40 \%$ incluster1and60\%incluster $2,80 \%$ incluster 1 and $20 \%$ in cluster $2,20 \%$ incluster 1 and $80 \%$ incluster 2,30\%incluster $\quad 1$ and70\%incluster $2,70 \%$ incluster 1 and $30 \%$ incluster $2,60 \%$ incluster

1 and $40 \%$ incluster $2,40 \%$ incluster 1 and60\%incluster(i.e.)between LOWandMODERATE.

Attributes 3,10,12,13,17,24 witha mean ratings 7.2,7.3,7.9,7.7,7.4,7.4 belongsto $80 \%$ incluster2and $20 \%$ incluster $3,70 \%$ incluster $\quad 2$ and $30 \% \quad$ incluster $3,10 \%$ incluster2and $90 \%$ incluster $3,30 \%$ in cluster2and70\%incluster3,60\%incluster 
2and40\%incluster3,60\%incluster2and betweenMODERATE and HIGH.

Table 3: Degree of Membership of the attributes Engineering faculties

\begin{tabular}{|c|c|c|c|c|}
\hline Attributes & Mean $_{2}$ & Low $_{2}$ & Moderate 2 & $\mathrm{High}_{2}$ \\
\hline 1 & 2.8 & 1 & 0 & 0 \\
\hline 2 & 8.7 & 0 & 0 & 1 \\
\hline 3 & 7.5 & 0 & 0.5 & 0.5 \\
\hline 4 & 9.1 & 0 & 0 & 1 \\
\hline 5 & 7.2 & 0 & 0.8 & 0.2 \\
\hline 6 & 6.7 & 0 & 1 & 0 \\
\hline 7 & 4.6 & 0.2 & 0.8 & 0 \\
\hline 8 & 8.2 & 0 & 0 & 1 \\
\hline 9 & 3.4 & 0.8 & 0.2 & 0 \\
\hline 10 & 7.4 & 0 & 0.6 & 0.4 \\
\hline 11 & 5.8 & 0 & 1 & 0 \\
\hline 12 & 3.8 & 0.6 & 0.4 & 0 \\
\hline 13 & 7.6 & 0 & 0.4 & 0.6 \\
\hline 14 & 5.3 & 0 & 1 & 0 \\
\hline 15 & 2.5 & 1 & 0 & 0 \\
\hline 16 & 7.3 & 0 & 0.7 & 0.3 \\
\hline 17 & 6.6 & 0 & 1 & 0 \\
\hline 18 & 7.7 & 0 & 0.3 & 0.7 \\
\hline 19 & 2.2 & 1 & 0 & 0 \\
\hline 20 & 3.2 & 0.9 & 0.1 & 0 \\
\hline 21 & 4.4 & 0.3 & 0.7 & 0 \\
\hline 22 & 6.4 & 0 & 1 & 0 \\
\hline 23 & 3.6 & 0.7 & 0.3 & 0 \\
\hline 24 & 7.6 & 0 & 0.4 & 0.6 \\
\hline 25 & 4.2 & 0.4 & 0.6 & 0 \\
\hline
\end{tabular}

Considering engineering faculties as the experts, degreeofmembership oftheattributesthat refer to the causes of failure in mathematics by engineering students isfoundusingtheabovealgorithm is showninTable: 3 .

Attributes 1,15,19witha meanrating2.8,2.5,2.2 isentirely (100\%)withamembership valueof 1 incluster 1. (i.e.)LOW.

Attributes6,11,14,17,22withameanrating6.7, 5.8, 5.3, 6.6, 6.4 isentirely(100\%)withamembershipvalue

of1incluster2.(i.e.)MODERATE.

Attribute $2, \quad 4,8$ withameanrating8.7, 9.1, 8.2,isentirely (100\%)with amembershipvalueof 1incluster 3. (i.e.)HIGH.

Attributes7,9,12,20,21,23,25withameanratings

4.6,3.4,3.8,3.2,4.4,3.6,4.2belongsto20\%incluster1

and

$80 \%$ incluster2,80\% incluster1 and $20 \%$ incluster $2,60 \%$ incluster 1and $40 \%$ in cluster $2,90 \%$ incluster1 and $10 \%$ incluster 2,30\%incluster 1 and $70 \%$ incluster $\quad 2, \quad 70 \%$ incluster 1and30\%incluster2,40\%incluster 1and60\%incluster2,

(i.e.)between LOWandMODERATE.

Attributes 3,5,10,13,16,18,24witha mean ratings 7.5,7.2,7.4,7.6,7.3,7.7,7.6 belongsto50\%incluster2and $50 \%$ incluster $3,80 \%$ incluster 2 and $20 \%$ incluster $3,60 \%$ incluster2and $40 \%$ incluster $3,40 \%$ in cluster2and60\%incluster3,70\%incluster 2and $30 \%$ incluster $3,30 \%$ incluster2and

$70 \%$ incluster3,40\%incluster2and $60 \%$ incluster3

betweenMODERATE and HIGH.

Table 4: Degree of Membership of the attributes -Parents of Engineering students

\begin{tabular}{|c|c|c|c|c|}
\hline Attributes & Mean $_{3}$ & Low $_{3}$ & Moderate 3 & High $_{3}$ \\
\hline 1 & 7.8 & 0 & 0.2 & 0.8 \\
\hline 2 & 6.2 & 0 & 1 & 0 \\
\hline 3 & 3.5 & 0.75 & 0.25 & 0 \\
\hline 4 & 7.5 & 0 & 0.5 & 0.5 \\
\hline 5 & 5.9 & 0 & 1 & 0 \\
\hline 6 & 4.6 & 0.2 & 0.8 & 0 \\
\hline 7 & 9.8 & 0 & 0 & 1 \\
\hline 8 & 2.7 & 1 & 0 & 0 \\
\hline 9 & 3.6 & 0.7 & 0.3 & 0 \\
\hline 10 & 6.6 & 0 & 1 & 0 \\
\hline 11 & 2.4 & 1 & 0 & 0 \\
\hline 12 & 7.4 & 0 & 0.6 & 0.4 \\
\hline 13 & 4.6 & 0.2 & 0.8 & 0 \\
\hline 14 & 6.2 & 0 & 1 & 0 \\
\hline 15 & 7.6 & 0 & 0.4 & 0.6 \\
\hline 16 & 3.6 & 0.7 & 0.3 & 0 \\
\hline 17 & 7.3 & 0 & 0.7 & 0.3 \\
\hline 18 & 9.4 & 0 & 0 & 1 \\
\hline 19 & 3.2 & 0.9 & 0.1 & 0 \\
\hline 20 & 2.2 & 1 & 0 & 0 \\
\hline 21 & 7.7 & 0 & 0.3 & 0.7 \\
\hline 22 & 6.7 & 0 & 1 & 0 \\
\hline 23 & 2.5 & 1 & 0 & 0 \\
\hline 24 & 7.9 & 0 & 0.1 & 0.9 \\
\hline 25 & 4.2 & 0.4 & 0.6 & 0 \\
\hline
\end{tabular}

Considering parents of engineering students as the experts, degreeofmembership oftheattributesthat refer to the causes of failure in mathematics by engineering students isfoundusingtheabovealgorithm is showninTable: 4

Attributes 8,11,20,23witha meanrating2.7, 2.4, 2.2, 2.5isentirely $(100 \%)$ withamembership valueoflincluster 1 . (i.e.)LOW.

Attributes2, 5, 10, 14, 22withameanrating6.2, 5.9, 6.6, 6.2, 6.7 isentirely $(100 \%)$ withamembershipvalue of1incluster2.(i.e.)MODERATE.

Attributes 7, 18withameanrating 9.8, 9.4isentirely (100\%)with amembershipvalue of 1 incluster 3 . (i.e.)HIGH.

\section{Attributes}

$3,6,9,13,16,19,25$ withameanratings $3 \cdot 5,4 \cdot 6,3 \cdot 6,4 \cdot 6,3 \cdot 6,3 \cdot 2,4.2 \mathrm{bel}$ ongsto75\%incluster 1

and $25 \%$ incluster $2,20 \%$ incluster1and $80 \%$ incluster $2,70 \%$ incluster 1 and $30 \%$ in cluster2,20\%incluster1and $80 \%$ incluster $2,70 \%$ incluster $\quad 1$ and30\%incluster $\quad 2$, 90\%incluster 1and10\%incluster2,40\%incluster 1 and60\%incluster2, (i.e.)between LOWandMODERATE. 
Attributes $1,4,12,15,17,21,24$ with a mean ratings 7.8,7.5,7.4,7.6,7.3,7.7,7.9 belongs to $20 \%$ in cluster 2 and $80 \%$ in cluster $3,50 \%$ in cluster 2 and $50 \%$ in cluster $3,60 \%$ in cluster 2 and $40 \%$ in cluster 3, $40 \%$ in cluster2and60\%incluster $3,70 \%$ in cluster2 and 30\% in cluster $3,30 \%$ in cluster 2 and $70 \%$ in cluster3, $10 \%$ in cluster2 and 90\%incluster3,(i.e.) between MODERATE and HIGH.

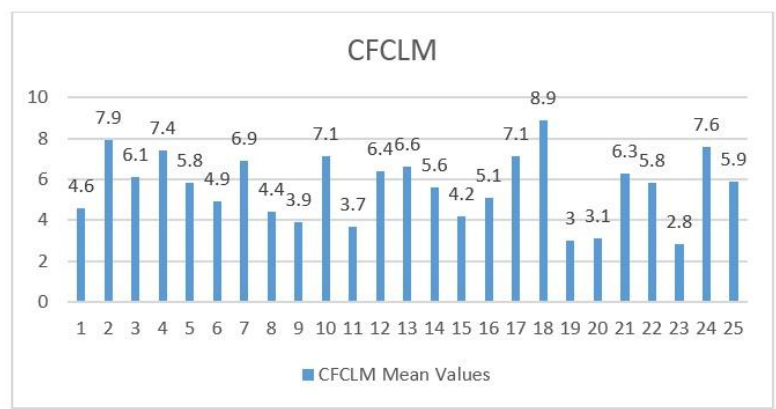

Fig 4:Mean rating of the Dominant cause- Combined Fuzzy Clustering Model

For the following Table 2, Table 3, and Table 4 Combined Fuzzy Clustering model is applied using the algorithm (5.2) and table CFCLM is obtained.

Combined degreeofmembership oftheattributesthat refer to the causes of failure in mathematics by engineering students isfoundusingtheabovealgorithm is showninTable 5: CFCLM and then thresholded and updated to obtain the on state and off state of the attributes among the three level of classifications, Low, Moderate and High.

On analysis attributes $1,8,9,11,15,19,20,23$ witha meanrating $4.6,4.4,3.9,3.7,4.2,3,3.1,2.8$ come to the 'on state' in cluster 1. (i.e.)LOW level.

Attributes $\quad 3,5,6,7,10,13,14,16,17,21,22,25$ withameanrating 6.1,5.8,4.9,6.9,7.1,6.6,5.6,5.1,7.1,6.3,5.8,5.9 come to the 'on state' incluster2.(i.e.)MODERATE level.

Attribute 2, 4, 12, 18, 24withameanrating 7.9, 7.4, 6.4, 8.9, 7.6 come to the 'on state'incluster 3. (i.e.)HIGH level.
Table 5: CFCLM Degree of Membership of the attributes Combined Fuzzy Clustering Model

\begin{tabular}{|c|c|c|c|c|c|c|c|c|}
\hline Attributes & Mean & Low & Moderate & High & $\longrightarrow$ & Low & Moderate & High \\
\hline 1 & 4.6 & 0.6 & 0.1 & 0.3 & $\longrightarrow$ & 1 & 0 & 0 \\
\hline 2 & 7.9 & 0 & 0.3 & 0.7 & $\longrightarrow$ & 0 & 0 & 1 \\
\hline 3 & 6.1 & 0.3 & 0.5 & 0.2 & $\longrightarrow$ & 0 & 1 & 0 \\
\hline 4 & 7.4 & 0 & 0.5 & 0.5 & $\longrightarrow$ & 0 & 0 & 1 \\
\hline 5 & 5.8 & 0.1 & 0.8 & 0.1 & $\longrightarrow$ & 0 & 1 & 0 \\
\hline 6 & 4.9 & 0.3 & 0.7 & 0 & $\longrightarrow$ & 0 & 1 & 0 \\
\hline 7 & 6.9 & 0.1 & 0.6 & 0.3 & $\longrightarrow$ & 0 & 1 & 0 \\
\hline 8 & 4.4 & 0.7 & 0 & 0.3 & $\longrightarrow$ & 1 & 0 & 0 \\
\hline 9 & 3.9 & 0.6 & 0.4 & 0 & $\longrightarrow$ & 1 & 0 & 0 \\
\hline 10 & 7.1 & 0 & 0.8 & 0.2 & $\longrightarrow$ & 0 & 1 & 0 \\
\hline 11 & 3.7 & 0.7 & 0.3 & 0 & $\longrightarrow$ & 1 & 0 & 0 \\
\hline 12 & 6.4 & 0.2 & 0.4 & 0.4 & $\longrightarrow$ & 0 & 0 & 1 \\
\hline 13 & 6.6 & 0.1 & 0.5 & 0.4 & $\longrightarrow$ & 0 & 1 & 0 \\
\hline 14 & 5.6 & 0 & 1 & 0 & $\longrightarrow$ & 0 & 1 & 0 \\
\hline 15 & 4.2 & 0.7 & 0.1 & 0.2 & $\longrightarrow$ & 1 & 0 & 0 \\
\hline 16 & 5.1 & 0.3 & 0.6 & 0.1 & $\longrightarrow$ & 0 & 1 & 0 \\
\hline 17 & 7.1 & 0 & 0.8 & 0.2 & $\longrightarrow$ & 0 & 1 & 0 \\
\hline 18 & 8.9 & 0 & 0.1 & 0.9 & $\longrightarrow$ & 0 & 0 & 1 \\
\hline 19 & 3 & 0.9 & 0.1 & 0 & $\longrightarrow$ & 1 & 0 & 0 \\
\hline 20 & 3.1 & 0.8 & 0.2 & 0 & $\longrightarrow$ & 1 & 0 & 0 \\
\hline 21 & 6.3 & 0.1 & 0.7 & 0.2 & $\longrightarrow$ & 0 & 1 & 0 \\
\hline 22 & 5.8 & 0.1 & 0.9 & 0 & $\longrightarrow$ & 0 & 1 & 0 \\
\hline 23 & 2.8 & 0.9 & 0.1 & 0 & $\longrightarrow$ & 1 & 0 & 0 \\
\hline 24 & 7.6 & 0 & 0.4 & 0.6 & $\longrightarrow$ & 0 & 0 & 1 \\
\hline 25 & 5.9 & 0.3 & 0.4 & 0.3 & $\longrightarrow$ & 0 & 1 & 0 \\
\hline & & & & & & & & \\
\hline
\end{tabular}

\section{CONCLUSION}

The Combined Fuzzy Clustering analysis highlighted thatamong twenty five attributes taken for analysis by the collective opinion from Engineering students, Engineering faculties and parents of engineering students we arrived the attributes $2,4,12,18,24$ (i.e)

- Parents join under compulsion in Engineering

- Higher secondary schools fail to provide clear knowledge in basics of XI and XII std syllabus

- Problem of language to understand mathematical terminology and the basics

- Providing only notes as study material than teaching

- Lack of interest in planning the time

are the major causes for the failures in mathematics by engineering students. These attributes resulted in on state after thresholding and updating in the high level of classification.

\section{SUGGESTION}

From the collective study of the experts, engineering students arecapable to overcome the other attributes to certain extent and get through the paper with minimum pass percentage. But the attributes resulting as dominant really should be taken steps by the government in regularizing the Educational system periodically, parents should jointheir children to their interest for their higher studies, Both Institution and Teacherscompulsorily take interest in giving thorough knowledge of XI and XII maths syllabus to the students, Engineering faculties have to involve themselves in teaching the subject rather than providing notes to the students, finally engineering students should realize the value of their studies. They should avoid wasting time due to varied reasons and utilize the time in coming regular to class, paying attention to the teaching, learning, and understanding, recalling the formulae and practicing the problems to get through the subject. 


\section{REFERENCES}

[1] A.Kaufmann, "Introduction to the Theory of Fuzzy Subsets", Academic Press ,INC.(LONDON) LTD,1975.

[2] B.Kosko, "Neural Networks and Fuzzy systems: A Dynamical System Approach to Machine Intelligence", Prentice Hall of India, 1997.

[3] H.J.Zimmermann, "Fuzzy Set Theory and its application", Fourth Edition Springer2011.

[4] J. Klir George/Bo Yuan, "FuzzysetsandFuzzy Logic: Theory and Applications", Prentice Hall of India.

[5] [5]VasanthaKandasamy W.B,Smarandache Florentin and K.Ilanthendral, "Elementary Fuzzy Matrix Theory and Fuzzy Models for Social Scientists"-2007.
[6] [6]A.VictorDevadoss,A.Felix, "FuzzyClusteringoftheDome sticViolencefor the Degree of Suicide thought based on Married Women Perception”,2012,IJERAp:1770-1773.

[7] [7]VasanthaKandasamy W.B and Smarandache Florentin "Fuzzy Cognitive Maps and Neutrosophic Cognitive Maps", Xiquan, Phoenix (2003).

[8] [8] W.B.Vasantha Kandasamy and S. Uma, "Combined Fuzzy Cognitive Maps of Socio EconomicModel"Appl.Sci.Periodical, 225-27(2000).

[9] [9]Fuzzy http://en.wikipedia.org/wiki/Fuzzy_clustering.

Clustering:

[10] [10]A.Victor Devadoss, A.Rajkumar and C.Jayalatha, "Analysis of Individual faith through Holy Bible Using Fuzzy Clustering”, IJCTE.2013.V5.785. 